\title{
Progress of Chinese and Western Medicine Clinical Research for Migraine
}

\author{
Dequan Liu ${ }^{1, a}$ Xiaoju Wang ${ }^{1, b}$ Jingjing Chang ${ }^{1, b}$ Shaoning Zhao ${ }^{1, d}$ Zhanpeng \\ Zhang $^{1, \mathrm{e}}$ Jing Zhang ${ }^{1, \mathrm{f}}$ \\ ${ }^{1}$ Langfang TCM Hospital, Langfang, Hebei, China, 065000 \\ ${ }^{a}$ email, ${ }^{b}$ email, ${ }^{c}$ email, ${ }^{d}$ email, ${ }^{e}$ email, ${ }^{f}$ email
}

Keywords: Migraine, Pathogenesis, Modern Medicine, Traditional Chinese Medicine

\begin{abstract}
Migrane is a common, chronic multifactorial disorders syndrome with multi-nervous system and non-nervous system disorder. The pathogens of migrane are still unclear, mind, diet, endocrine, heredity have been considered to be attributed to it. Pathogenesis and therapeutic have been explored constantly. Now, an effective migraine modern medicine is based on non-pharmacological treatment as well as acute and preventive medication. Traditional Chinese medicine has been developed day by day, which will establish new directions for migrainous research and therapeutic.
\end{abstract}

\section{Introduction}

Migraine is a clinically common causes of complex, easy repeatedly, seriously affecting a class of neurovascular disease of people's lives, to one or both sides of the head pain, accompanied by nausea, vomiting as the main clinical features, a few typical before the onset of seizures in patients with visual, sensory, motor and other threatened, has a family history. All countries reported prevalence of women was 3.3\% 32.6\% for men and $0.7 \% \sim 16.1 \%$. World Health Organization (WHO) 2001 set most severely disabling migraine as a chronic disease, similar to dementia, paralysis of the limbs and severe mental illness. Migraine causes complex, modern medical research and treatment status of its pathogenesis has made some achievements. Migraine is the medicine first wind, wind brain, headache and other areas, is fully wind, phlegm, silt and other factors together, Chinese medicine in the treatment of migraine on the advantages become increasingly evident. This paper will study recent migraine before reviewed.

\section{Pathogenesis}

Neurogenic Theory. the theory of the more important hypotheses neurotransmitter theory and cortical spreading inhibition (cortical spreading depression CSD) doctrine. The study found some impact on CSD subcortical structures such as the thalamus, the spinal trigeminal nucleus, possibly CSD activities like nerve points cause headaches and clinical manifestations of the important reasons.

Angiogenic Theory. Early studies suggest that aura and migraine without aura are differences in the degree of vascular spasm of the same disease. Recent studies have found that some patients with migraine attacks decreased cerebral blood flow, cerebral blood flow in patients also have a small number of patients and even increase after the first decrease, and these changes are not without aura constant relationship. The relationship between migraine angiogenic and doctrine cerebrovascular dysfunction needs to be confirmed by further studies.

Biochemical Theory. The vasoactive substances currently the most talked about are serotonin (5-HT), calcitonin gene-related peptide (CGRP), dopamine, ON and so on. Ischemia, mental stress, fatigue or other factors can lead to a migraine attack, different vasoactive receptor agonist, antagonist effective application in migraine, and further illustrates the variation of its existence and rationality, and for the development of migraine drugs provides new directions.

Genetics Theory. The clinical study found that migraine familial aggregation tendency, and the 
same family line may occur simultaneously with aura and migraine without aura, suggesting that they may have common genetic mechanism. Familial hemiplegic migraine (FHM) is a genetic factor apparent migraine with aura (MA), its mode of inheritance of single gene, autosomal dominant inheritance. Three known genes associated with: CACNA1A, ATP1A2 and SCN1A.

Traditional Chinese Medical. The headache contained in a license first "via", called "first wind" and "Brain wind" in the "Q • wind theory", exogenous and internal injuries are an important cause of headache. Wind, cold, fire, phlegm, silt migraine onset disease group, and associated with the liver, spleen, kidney three dirty. Chinese medicine believes that the head for Zhu Yang will, the blood will be on top of the roof are. If the wind evil on the disturbance and be blocked and it can be made for the headache; study found that most recurrent migraine, the "wind phlegm and blood" of the characteristics, and the relationship with the liver most closely.

\section{Prevention and Prophylaxis}

Prevention: Note patterns of life, to avoid fatigue, stress, prevention and treatment of sub-health status. Identify and alleviate factors that induce headaches and try to avoid.

Migraine preventive treatment should be individualized, particularly in individual doses. You can select the initial dose based on the patient's physical condition, past experience and other medication, gradually increase the amount, if no significant adverse reactions, can be used in conjunction with a few days, followed by other medications fail. Common preventive medicine include: $\beta$-blockers, calcium channel blockers, antiepileptic agents, antidepressants, NSAID and other species (TCM) pharmaceutical all can be tried.

\section{Treatment}

Basic principles: to strengthen patient education guide; take advantage of a variety of non-pharmacological interventions; drug treatment if necessary.

Patient Education. Migraine is currently no cure but can be controlled effectively. To help them establish a scientific and rational prevention concepts and objectives; to educate patients maintain a healthy lifestyle, learn to look for and avoid all kinds of headache triggers; encourage patients to remember a headache diary to help diagnose and assess treatment is important to prevent.

Modern Medicine Drug Treatment. Acute exacerbation of drug therapy treatment goals: fast pain relief, short-term to avoid relapse, improve the body's cognitive function, reduce the waste of medical resources. Drug use should be a headache in early enough to use, with severe nausea and vomiting, should be selected for parenteral administration. metoclopramide, domperidone and other antiemetic and promote gastric motility drugs not only to treat associated symptoms, but also help to absorb and headaches treatment of other drugs.

The pharmaceutical characteristics of acute exacerbation of pharmaceutical drugs are divided into non-specific and specific drug therapy. Non-specific drugs include: (1) non-steroidal anti-inflammatory drugs (NSAIDs), including acetaminophen, aspirin, ibuprofen and its compound preparation; (2) codeine, tramadol and morphine and other opiates analgesics; (3) barbiturates and other sedatives. The first two drugs when used should be strictly controlled indications, only apply to other cases of therapy, the latter two drugs can quickly relieve symptoms, but easily lead to more frequent episodes and addiction, it should be used with caution. Specific drugs include: (1) ergot preparations, mainly refers ergotamine caffeine; (2) there are triptans sumatriptan, zolmitriptan, rizatriptan, etc; (3) Calcitonin gene-related peptide receptor antagonist (CGRP).

Application of Chinese Medicine. The syndrome differentiation is the soul of traditional Chinese medicine, Chinese medicine treatment on the basis that all differentiation, differentiation by many doctors who use Chinese classic prescription migraine treatment in the clinic has achieved remarkable results. Such as: Modified Banxiabaizhutianma soup, Danzhixiaoyao Powder, Evodia soup flavored and other classic migraine prescriptions in the field of successful application. In the dosage form, in addition to the traditional Chinese medicine decoction, but also developed a proprietary compound medicine and injections. Chinese medicine treatment of migraine in the 
overall concept as a guide, using the principles of diagnosis and treatment, not only drug therapy, more acupuncture, massage, acupuncture therapy and other external treatment.

\section{Acknowledgements}

Fund Project: Langfang City Science and Technology Research and Development Plan Program (2013013014C)

\section{References}

[1] Zhang LM1, Dong Z1, Yu SY1, et al. Ann Transl Med, Vol. 6 (2016) No 4, p.105

[2] Martins IP. Migraine. Acta Mad Port, Vol. 5 (2009) No 22, p. 589-598

[3] Dichgans M, Freilinger T, Eckstein G, et al. Lancet, Vol. 366 (2005) No9483, p. 371-377

[4] Wang Wei, Quan Yaping, Chen Yuantao. Changchun University of Traditional Chinese Medicine, Vol. 2 (2013) No 29, p. 262-263

[5] Kong Fanxia, Fan Daochang. Sichuan Traditional Chinese Medicine, Vol. 9 (2002) No 20, p.38 